\title{
Influence of surface displacement on solid state flow induced by horizontally heterogeneous Joule heating in the inner core of the Earth
}

\author{
$\operatorname{AUTHOR(S):~}$ \\ Takehiro, Shin-ichi
}

\section{CITATION:}

Takehiro, Shin-ichi. Influence of surface displacement on solid state flow induced by horizontally heterogeneous Joule heating in the inner core of the Earth. Physics of the Earth and Planetary Interiors 2015, 241: 15-20

\section{ISSUE DATE:}

2015-04

URL:

http://hdl.handle.net/2433/196749

\section{RIGHT:}

(c) 2015 Elsevier B.V. NOTICE: this is the author's version of a work that was accepted for publication in Physics of the Earth and Planetary Interiors. Changes resulting from the publishing process, such as peer review, editing, corrections, structural formatting, and other quality control mechanisms may not be reflected in this document. Changes may have been made to this work since it was submitted for publication. A definitive version was subsequently published in Physics of the Earth and Planetary Interiors, 241, doi:10.1016/.j.pepi.2015.02.003; この論文は出版社版でありません。引 用の際には出版社版をご確認ご利用ください。; This is not the published version. Please cite only the published version. 


\title{
Influence of surface displacement on solid state flow induced by horizontally heterogeneous Joule heating in the inner core of the Earth
}

\author{
Shin-ichi Takehiro \\ Research Institute for Mathematical Sciences, Kyoto University, Sakyo-ku, Kyoto 606-8502, Japan
}

\begin{abstract}
We investigate the influence of surface displacement on fluid motions induced by horizontally heterogeneous Joule heating in the inner core. The difference between the governing equations and those of Takehiro (2011) is the boundary conditions at the inner core boundary (ICB). The temperature disturbance at the ICB coincides with the melting temperature, which varies depending on the surface displacement. The normal component of stress equalizes with the buoyancy induced by the surface displacement. The toroidal magnetic field and surface displacement with the horizontal structure of $Y_{2}^{0}$ spherical harmonics is given. The flow fields are calculated numerically for various amplitudes of surface displacement with the expected values of the parameters of the core. Further, by considering the heat balance at the ICB, the surface displacement amplitude is related to the turbulent velocity amplitude in the outer core, near the ICB. The results show that when the turbulent velocity is on the order of $10^{-1}-10^{-2}$ $\mathrm{m} / \mathrm{s}$, the flow and stress fields are similar to those of Takehiro (2011), where the surface displacement vanishes. As the amplitude of the turbulent velocity decreases, the amplitude of the surface displacement increases, and counter flows from the polar to equatorial regions emerge around the ICB, while flow in the inner regions is directed from the equatorial to polar regions, and the non-zero radial component of velocity at the ICB remains. When the turbulent velocity is on the order of $10^{-4}-10^{-5} \mathrm{~m} / \mathrm{s}$, the radial component of velocity at the ICB vanishes, the surface counter flows become
\end{abstract}

Email address: takepiro@gfd-dennou.org (Shin-ichi Takehiro) 
stronger than the flow in the inner region, and the amplitude of the stress field near the ICB dominates the inner region, which might be unsuitable for explaining the elastic anisotropy in the inner core.

Keywords: Inner core flows, Elastic anisotropy, Turbulent velocity in the outer core, Interaction between inner and outer core

\section{Introduction}

The origin of the elastic anisotropy of the Earth's inner core (e.g. Poupinet et al., 1983; Morelli et al., 1986; Souriau, 2007) is considered to be the alignment of texture formed along the solidification of the core (e.g. Karato, 1993; Bergman, 1997) or the alignment of the preferred orientation of crystals by plastic deformation of fluid motions (e.g. Jeanloz and Wenk, 1988; Yoshida et al., 1996; Karato, 1999; Buffett and Wenk, 2001). The depth dependency of the anisotropy is difficult to explain by the solidification mechanism, whereas the various factors driving solid state flow in the inner core considered thus far do not appear to yield sufficiently strong stresses to generate elastic anisotropy. Takehiro (2011) proposed Joule heating of the magnetic field penetrating diffusively from the inner core boundary (ICB) as a possible source of inner core flows. His specific calculation in the case of a toroidal magnetic field with the horizontal structure of $Y_{2}^{0}$ spherical harmonics showed that internal flows of sufficient magnitude can be induced to explain the elastic anisotropy. The obtained solution consists of downward flow in the equatorial region and upward flows in the polar region, and has a non-zero radial velocity component at the ICB, causing mass exchange between the inner and outer core. This feature is a result of the constant normal stress boundary condition at the ICB, and it is implicitly assumed that the phase change occurs instantaneously at the ICB. However, the actual speed of the phase change is finite. If the speed of the phase change is slow enough, the ICB would be deformed, and surface displacement is induced by the non-zero radial velocity at the ICB. This surface displacement may prevent inner core flows due to the buoyancy force originating from the density contrast between the inner and outer core.

In this paper, we investigate the influence of surface displacement on fluid motions 
induced by horizontally heterogeneous Joule heating in the inner core. We examine the extent of development of surface displacement, and modification of the flow field of the inner core. Sec. 2 is a description of our model. In Sec. 3, numerical results

We consider an MHD Boussinesq fluid in a sphere. The governing equations deter-

mining steady flow and temperature disturbance induced by differential Joule heating are as follows (Takehiro, 2011):

$$
\begin{aligned}
& 0=-\frac{1}{\rho_{0}} \nabla p+\alpha T \boldsymbol{g}+\nu \nabla^{2} \boldsymbol{v}, \\
& v_{r} \frac{d T_{B}}{d r}=\kappa \nabla^{2} T+\frac{Q_{J}}{\rho_{0} C_{p}}, \\
& \nabla \cdot \boldsymbol{v}=0 .
\end{aligned}
$$

${ }_{37} \boldsymbol{v}$ is velocity, $v_{r}$ is the radial component of velocity, $\rho_{0}$ is the mean density of the 38 Boussinesq fluid, $p$ is pressure, $T$ is the temperature disturbance, and $d T_{B} / d r$ is the raз9 dial temperature gradient of the basic state. Gravity induced by the mass of the sphere 40 itself is a spherically symmetric distribution, $\boldsymbol{g}=-\left(g_{0} / a\right) \boldsymbol{r}$, where $g_{0}$ is the gravita${ }_{41}$ tional acceleration at the surface, $a$ is the radius of the sphere, and $\boldsymbol{r}$ is the position 42 vector in the radial direction. $Q_{J}=|\boldsymbol{J}|^{2} / \sigma=|\nabla \times \boldsymbol{B}|^{2} / \mu \sigma$ is the Joule heating produced ${ }_{43}$ by the magnetic field $\boldsymbol{B}$ diffusing from the outer boundary (ICB) to the interior, where ${ }_{44} \mu$ and $\sigma$ are the magnetic permeability and electric conductivity. Note that eqs. (1) 45 and (2) neglect second order nonlinear terms, the validation of which was discussed in 46 Takehiro (2011).

${ }_{47}$ The difference between these governing equations and those of Takehiro (2011) is 48 the boundary conditions at the ICB, where the effects of surface displacement emerge.

49 The normal stress is balanced at the surface with a buoyancy force proportional to the 
density difference of the inner and outer core. The temperature at the surface is equal to the melting point, which is varied by the surface displacement. The tangential stresses vanish at the surface.

$$
\begin{aligned}
& \sigma_{r r}=-p+2 \rho_{0} v \frac{\partial v_{r}}{\partial r}=-\Delta \rho g h \\
& \sigma_{r \theta}=\rho_{0} v\left(\frac{1}{r} \frac{\partial v_{r}}{\partial \theta}+\frac{\partial v_{\theta}}{\partial r}-\frac{v_{\theta}}{r}\right)=0, \sigma_{r \phi}=\rho_{0} v\left(\frac{\partial v_{\phi}}{\partial r}-\frac{v_{\phi}}{r}+\frac{1}{r \sin \theta} \frac{\partial v_{r}}{\partial \phi}\right)=0 \\
& T=\frac{d T_{m}}{d r} h, \quad \text { at } \quad r=a
\end{aligned}
$$

53 Here, $\Delta \rho$ is the density difference between the inner and outer core, $h(\theta, \phi)$ is the surface

54 displacement distribution, $\theta$ and $\phi$ are colatitude and azimuth, respectively, and $d T_{m} / d r$

55 is the melting temperature gradient. For simplicity, stress and temperature are evaluated

56 at $r=a$, which is the boundary where the surface displacement vanishes.

57 The non-divergent flow field is expressed with the toroidal and poloidal potentials,

${ }_{58} \psi$ and $\Phi$, defined by

$$
\boldsymbol{v}=\nabla \times(\psi(r, \theta, \phi) \boldsymbol{r})+\nabla \times \nabla \times(\Phi(r, \theta, \phi) \boldsymbol{r}),
$$

59 Eqs. (1) and (2) become

$$
\begin{aligned}
& \nabla^{2} L_{2} \psi=0, \\
& v \nabla^{2} L_{2} \nabla^{2} \Phi-\alpha\left(g_{0} / a\right) L_{2} T=0, \\
& \frac{L_{2} \Phi}{r} \frac{d T_{B}}{d r}=\kappa \nabla^{2} T+\frac{Q_{J}}{\rho_{0} C_{p}} .
\end{aligned}
$$

60 From Eq. (8), $\psi \equiv 0$, meaning that the toroidal component is not induced. Removing

61 the temperature disturbance from Eqs. (9) and (10),

$$
\frac{L_{2} \Phi}{r} \frac{d T_{B}}{d r}-\frac{\kappa v}{\alpha\left(g_{0} / a\right)} \nabla^{2} \nabla^{2} \nabla^{2} \Phi=\frac{Q_{J}}{\rho_{0} C_{p}} .
$$

62 The boundary conditions are expressed with the velocity potentials. By taking the

63 horizontal divergence of Eq. (1), pressure can be expressed with the potentials. Then,

64 Eqs. (4), (5), and (6) become

$$
\begin{aligned}
& \rho_{0} v \frac{\partial}{\partial r} r\left(-\nabla^{2} \Phi+\frac{2 L_{2} \Phi}{r^{2}}\right)=-\Delta \rho g h \text { at } r=a, \\
& \frac{\partial^{2} \Phi}{\partial r^{2}}-\frac{2 \Phi}{r^{2}}+\frac{L_{2} \Phi}{r^{2}}=0 \text { at } r=a, \\
& \frac{v a}{\alpha g_{0}} \nabla^{2} \nabla^{2} \Phi=\frac{d T_{m}}{d r} h, \text { at } r=a .
\end{aligned}
$$


Following the procedure of Takehiro (2011), the governing equations are nondimensionalised, considering the dominance of advection of basic temperature. Using

67 the temperature rising rate $\left|Q_{J}\right| / \rho C_{p}$ and the difference between basic and adiabatic

68 temperature at the center, $\Delta T$, the time scale is chosen to be $\Delta T \rho C_{p} /\left|Q_{J}\right|$. The length scale is chosen to be the radius of the sphere $a$. Then, the poloidal potential should be 70 normalised by $\left(\left|Q_{J}\right| / \rho C_{p}\right)\left(a^{2} / \Delta T\right)$. Eq. (11) becomes

$$
\frac{L_{2} \Phi}{r} \frac{d T_{B}}{d r}-\frac{1}{R} \nabla_{2} \nabla_{2} \nabla_{2} \Phi_{*}=q_{J},
$$

${ }_{71} \quad$ where $q_{j}=Q_{J} /\left|Q_{J}\right|$ is non-dimensionalised Joule heating, and $R$ expresses the strength

72 of stable stratification,

$$
R=\frac{\alpha g_{0} \Delta T a^{3}}{\kappa v} .
$$

73 The boundary conditions, Eqs. (12), (13), and (14) are normalised as:

$$
\begin{aligned}
& \frac{\partial}{\partial r} r\left(-\nabla^{2} \Phi+\frac{2 L_{2} \Phi}{r^{2}}\right)=-R_{s} h, \text { at } r=1, \\
& \frac{\partial^{2} \Phi}{\partial r^{2}}-\frac{2 \Phi}{r^{2}}+\frac{L_{2} \Phi}{r^{2}}=0 \text { at } r=1, \\
& \frac{1}{R} \nabla^{2} \nabla^{2} \Phi=-\Gamma_{m} h, \text { at } r=1,
\end{aligned}
$$

74 where

$$
\Gamma_{m}=\frac{\left(-d T_{m} / d r\right) a}{\Delta T}=\frac{\left(d T_{m} / d P\right) \rho g a}{\Delta T}, \quad R_{s}=\frac{\rho C_{p} \Delta T}{\left|Q_{J}\right|} \frac{\Delta \rho g a}{\rho_{0} v} .
$$

Given the values of $R, \Gamma_{m}$, and $R_{s}$, the steady flow and temperature disturbance fields can be obtained from these equations by setting the distributions of basic temperature gradient $d T_{B} / d r$, Joule heating $q_{J}$, and surface displacement $h$.

To solve the governing equations with the boundary conditions numerically, the poloidal potential $\Phi$ is expanded with spherical harmonic functions in the horizontal directions, and with the polynomials developed by Matsushima and Marcus (1995) in the radial direction. The surface displacement $h$ is also expanded with spherical harmonics. Then, the problem becomes a system of linear equations for each spherical harmonic component of $\Phi$, since the governing equations and boundary conditions are linear. The polynomials for the radial direction are calculated to the 63rd degree.

In the same manner as the specific calculation of Takehiro (2011), the toroidal magnetic field component with spherical harmonics of degree 2 and order 0 is imposed 


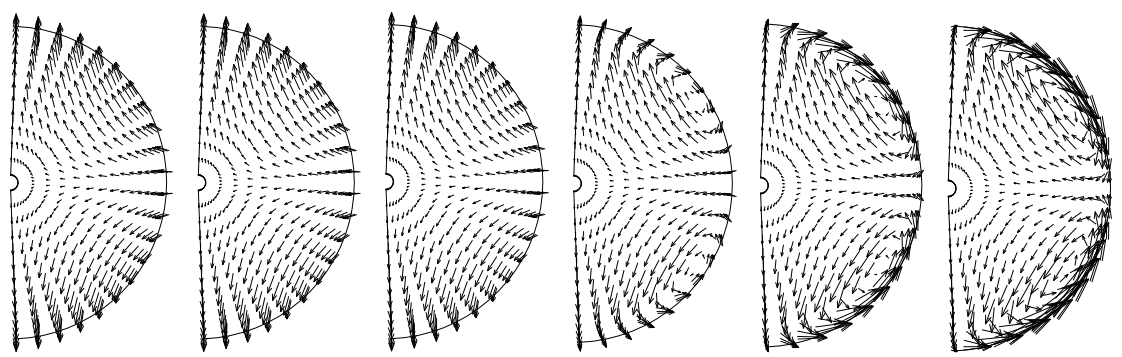

Figure 1: Flow fields in the inner core induced by Joule heating of $Y_{2}^{0}$ type for various amplitudes of surface displacement. From left to right, given amplitudes of surface displacement that are 0, 0.006, 0.06, 0.6, 1.2, and $1.8 \mathrm{~m}$ in the case of $B=10^{-1} \mathrm{~T}$ (or 0, 0.00006, $0.0006,0.006,0.012$, and $0.018 \mathrm{~m}$ in the case of $B=10^{-2}$ T). These panels correspond to cases with turbulent velocities in the outer core of $u^{\prime} \sim \infty, 1.4 \times 10^{-1}, 1.4 \times$ $10^{-2}, 10^{-3}, 2.6 \times 10^{-4}$, and $2.1 \times 10^{-5} \mathrm{~m} / \mathrm{s}$, respectively. on the ICB. The Joule heating distribution in the inner core produced by the steady magnetic field diffusing from the ICB becomes $q_{J}=r^{2} Y_{2}^{0}(\cos \theta)$, removing the homogeneous component, and its amplitude is given by $\left|Q_{J}\right|=8 B^{2} /\left(\sigma \mu^{2} a^{2}\right)$ (Takehiro, 2011). Since the governing equations of the system are linear, the surface displacement distribution at the ICB induced by the flow driven by Joule heating is also proportional to $Y_{2}^{0}$. Following the setup of Takehiro (2011), the non-dimensionalised temperature gradient of the basic state $d T_{B} / d r$ is assumed to be in proportion to $r$. Table 1 summarizes the values of the parameters used for the numerical calculations. Note that larger values of electric conductivity and thermal diffusivity recently estimated by first principle calculations (e.g. Pozzo et al., 2012) are adopted than those used in Takehiro

97 (2011). Using these values, the non-dimensional parameters are estimated as:

$$
\begin{aligned}
& R=\frac{\alpha g \Delta T a^{3}}{\kappa v} \sim 1.6 \times 10^{7}, \quad R s=\frac{\rho C_{p} \Delta T}{Q_{J}} \frac{\Delta \rho g a}{\rho_{0} v} \sim 3.1 \times 10^{8}-3.1 \times 10^{10}, \\
& \Gamma_{m}=\frac{m_{m} \rho g a}{\Delta T} \sim 20.4 .
\end{aligned}
$$

\section{3. Results}

Fig. 1 shows the obtained flow field for several amplitudes of surface displacement. When the magnitude of magnetic field at the ICB is $B=10^{-1} \mathrm{~T}$, the distribution 


\begin{tabular}{|c|r|}
\hline Magnetic field at ICB $B$ & $10^{-1}-10^{-2} \mathrm{~T}$ \\
Electric conductivity $\sigma$ & $1.2 \times 10^{6} \mathrm{Sm}$ \\
Magnetic permeability $\mu$ & $4 \pi \times 10^{-7}$ \\
Inner core radius $a$ & $1.2 \times 10^{6} \mathrm{~m}$ \\
Inner core density $\rho_{0}$ & $1.2 \times 10^{4} \mathrm{~kg} / \mathrm{m}^{3}$ \\
Density difference between inner and outer core $\Delta \rho$ & $5 \times 10^{2} \mathrm{~kg} / \mathrm{m}^{3}$ \\
Specific heat $C_{p}$ & $850 \mathrm{~J} / \mathrm{kg} \cdot \mathrm{K}$ \\
Gravity at ICB $g$ & $5 \mathrm{~m} / \mathrm{s}^{2}$ \\
$30 \mathrm{~K}$ \\
Difference between basic and adiabatic temperature at the center $\Delta T$ \\
Thermal expansion coefficient $\alpha$ & $1 \times 10^{-5} 1 / \mathrm{K}$ \\
Thermal diffusivity $\kappa$ & $2 \times 10^{-5} \mathrm{~m} / \mathrm{s}$ \\
Viscosity & $10^{17} \mathrm{~Pa} \cdot \mathrm{s}$ \\
Latent heat $L$ & $10^{6} \mathrm{~J} / \mathrm{kg}$ \\
Adiabatic temperature gradient near ICB $m_{a d}$ & $6 \times 10^{-9} \mathrm{~K} / \mathrm{Pa}$ \\
Melting temperature gradient near ICB $m_{m}$ & $8.5 \times 10^{-9} \mathrm{~K} / \mathrm{Pa}$ \\
Turbulent velocity near ICB in the outer core $u^{\prime}$ & $10^{-1}-10^{-5} \mathrm{~m} / \mathrm{s}$ \\
\hline
\end{tabular}

Table 1: Values of inner core model parameters used for numerical calculations. Physical properties of the inner core are from Stacey and Davis (2008), $\sigma$ and $\kappa$ are from Pozzo et al. (2012), $m_{a d}$ and $m_{m}$ are from Alboussiere et al. (2010), and $u^{\prime}$ is from Loper (2007). 
of the flow field in the case with a surface displacement magnitude of $0.006-0.06 \mathrm{~m}$ is similar to the case of no surface displacement. The fact that the surface displacement does not affect significantly to the fluid field means that timescale of phase change at ICB is small compared with that of surface deformation in these cases. Recalling the result of Takehiro (2011), the solid state flow is mainly driven so that temperature increase/decrease by heterogenous Joule heating balances with the advection of the basic temprature. As a result, the flow velocity is essentially independent of the viscosity of the inner core. In other words, since the timescale of advection of temperature disturbance is small compared to that of advection of basic temperature, the inner core continuously deforms to keep the isotherms as close as possible to spherical surfaces. The amplitude of induced solid state flow is $O\left(10^{-10}\right) \mathrm{m} / \mathrm{s}$, which is smaller than the estimation by Takehiro (2011) due to the larger value of electric conductivity. As the amplitude of surface displacement increases to $O(1 \mathrm{~m})$, the counter flow from the poles to the equator emerges, and is strengthened below the ICB. However, in the deep region, the flows directed from the equator to the poles still exist, and the magnitude of the internal flows is similar to the case with no surface displacement. The normal component of velocity at the ICB vanishes when the amplitude of surface displacement is about $1.8 \mathrm{~m}$, where the amplitude of surface velocity becomes $3 \times 10^{-10} \mathrm{~m} / \mathrm{s}$. As the ICB approaches to a closed boundary, the amplitude of flow below the ICB increases, because mass flux from the equatorial to the polar regions by the deep flows (which does not change its amplitude) must return through the thin layer below the ICB. Fig. 2 shows the direction and magnitude of the principal stresses of the flow fields presented in Fig. 1. When the magnitude of the magnetic field at the ICB is $B=10^{-1} \mathrm{~T}$, the distribution of the stress field in the case where the amplitude of surface displacement of $0.006 \mathrm{~m}$ is similar to the case of no surface displacement, Its magnitude is $O(10) \mathrm{Pa}$, which is smaller than the estimation by Takehiro (2011) due to the larger value of electric conductivity. The principal stress below the ICB is weak and directed in a different direction from that in the deep region. As the amplitude of surface displacement increases to $O(1 \mathrm{~m})$, the magnitude of principal stress below the ICB becomes as large as $O\left(10^{2}\right) \mathrm{Pa}$ and its direction is parallel to the equatorial plane. However, in the deep region, the principal stress keeps its magnitude and is directed poleward, which is the 


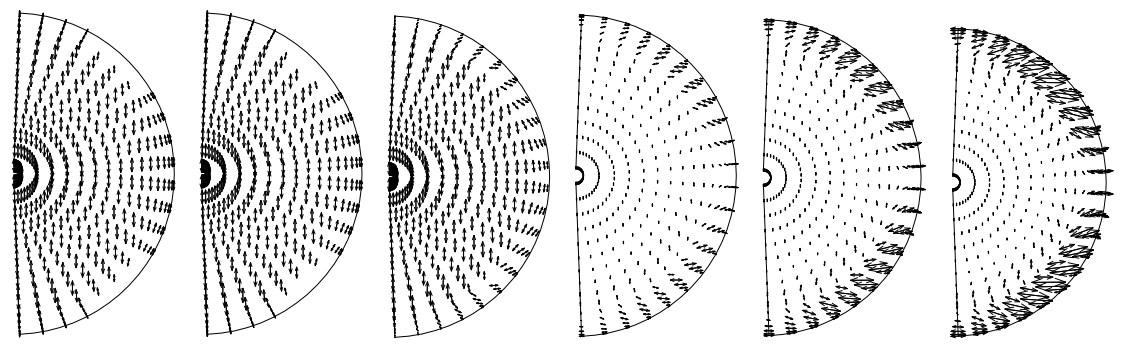

Figure 2: Direction and magnitude of principal stresses of the flow fields presented in Fig. 1. From left to right, given amplitudes of surface displacements that are $0,0.006,0.06,0.6,1.2$, and $1.8 \mathrm{~m}$ in the case of $B=$ $10^{-1} \mathrm{~T}$ (or $0,0.00006,0.0006,0.006,0.012$, and $0.018 \mathrm{~m}$ in the case of $B=10^{-2} \mathrm{~T}$ ). These panels correspond to cases with turbulent velocities in the outer core of $u^{\prime} \sim \infty, 1.4 \times 10^{-1}, 1.4 \times 10^{-2}, 10^{-3}, 2.6 \times 10^{-4}$, and $2.1 \times 10^{-5} \mathrm{~m} / \mathrm{s}$, respectively. The scale of the arrows in the three right panels are $1 / 5$ of the arrows in the three left panels. 143 heat for melting. Then, we have

$$
u^{\prime}=\frac{v_{r}(r=a) L}{C_{p}\left(m_{m}-m_{a d}\right) \rho g h} .
$$

$$
u^{\prime} C_{p} \delta T \sim u^{\prime} C_{p}\left(m_{m}-m_{a d}\right) \rho g h,
$$

where $\delta T$ is the adiabatic and melting temperature difference, and $m_{m}$ and $m_{a d}$ are the melting and adiabatic temperature gradients near the ICB, respectively. This heat transport should be balanced by the latent heat for melting of the solid material ejected from the ICB, $v_{r}(r=a) L$, where $v_{r}(r=a)$ is radial flow at the ICB, and $L$ is the latent

$u^{\prime}$ are evaluated by using the numerical results of $v_{r}(r=a)$ and $h$. Table 2 shows the values of turbulent velocity in the outer core $u^{\prime}$ for various values of the amplitudes of surface displacement and radial flow at the ICB. When the turbulent velocity is 


\begin{tabular}{c|cccccc}
$h(\mathrm{~m})$ & 0 & 0.006 & 0.06 & 0.6 & 1.2 & 1.8 \\
$v_{r}(r=a)(\mathrm{m} / \mathrm{s})$ & $1.1 \times 10^{-10}$ & $1.1 \times 10^{-10}$ & $1.1 \times 10^{-10}$ & $7.6 \times 10^{-11}$ & $4.0 \times 10^{-11}$ & $4.9 \times 10^{-12}$ \\
\hline$u^{\prime}(\mathrm{m} / \mathrm{s})$ & $\infty$ & $1.4 \times 10^{-1}$ & $1.4 \times 10^{-2}$ & $10^{-3}$ & $2.6 \times 10^{-4}$ & $2.1 \times 10^{-5}$
\end{tabular}

Table 2: Turbulent velocity in the outer core $u^{\prime}$ calculated with equilibrium amplitudes of surface displacement and radial flow at the ICB in the case of $B=10^{-1} \mathrm{~T}$.

sufficiently large $\left(u^{\prime} \sim 10^{-1}-10^{-2} \mathrm{~m} / \mathrm{s}\right)$, the amplitude of surface displacement becomes small $\left(h \sim 10^{-2} \mathrm{~m}\right)$, since the growth time scale of surface displacement is large enough compared to the time scale of phase change. Then, the velocity and stress fields in the inner core are similar to those in the case of no surface displacement (the left three panels of Figs. 1 and 2$)$. In contrast, when the turbulent velocity is small $\left(u^{\prime} \sim 10^{-3}-\right.$ $10^{-5} \mathrm{~m} / \mathrm{s}$ ), the surface displacement becomes as large as $h \sim 1 \mathrm{~m}$, since the growth time scale of surface displacement is small compared to the time scale of phase change. Then, the radial flows at the ICB are weakened, and strong return flows from the poles to the equator emerge near the ICB, while flows from the equator to the poles with amplitudes similar to the $h=0$ case remain in the interior (the right three panels of Figs. 1 and 2).

\section{Conclusions and discussions}

We investigated the fluid motions induced by horizontally heterogeneous Joule heating in the inner core by taking into account the surface displacement. Given an ICB toroidal magnetic field of $Y_{2}^{0}$ type, the distributions of the flow and stress fields were calculated for various values of surface displacement amplitude. Further, the relationship between the amplitudes of surface displacement and radial flow at the ICB was deduced from the heat balance at the ICB, and, as a result, the distributions of the flow and stress fields were obtained for various values of turbulent velocity near the ICB in the outer core. The results show that when the turbulent velocity is sufficiently large $\left(u^{\prime} \sim 10^{-1}-10^{-2} \mathrm{~m} / \mathrm{s}\right)$, the surface displacement does not develop significantly, and the velocity and stress fields in the inner core are similar to those in the case of no surface displacement (the left three panels of Figs. 1 and 2), which may explain the elastic anisotropy, although those magnitudes are estimated as $O\left(10^{-10}\right) \mathrm{m} / \mathrm{s}$ and 
$O(10) \mathrm{Pa}\left(O\left(10^{-12}\right) \mathrm{m} / \mathrm{s}\right.$ and $O(0.1) \mathrm{Pa}$ for $\left.B=10^{-2} \mathrm{~T}\right)$, which are smaller than the estimation by Takehiro (2011) due to the smaller value of electric conductivity adopted here. In contrast, when the turbulent velocity is small $\left(u^{\prime} \sim 10^{-3}-10^{-5} \mathrm{~m} / \mathrm{s}\right)$, the rate of phase change decreases at the ICB, and the surface displacement develops significantly. The radial flows at the ICB is weakened, and strong return flows from the poles to the equator emerge near the ICB (the right three panels of Figs. 1 and 2), which may not be suitable for explaining the origin of anisotropy in the inner core. These results suggest that the amplitude of turbulent velocity in the outer core should be as large as $u^{\prime} \sim 10^{-2} \mathrm{~m} / \mathrm{s}$ in order to attribute the origin of anisotropy in the inner core to the fluid motions induced by heterogeneous Joule heating.

The amplitude of turbulent velocity in the outer core is considered to be on the order of $10^{-3} \mathrm{~m} / \mathrm{s}$ or $10^{-4} \mathrm{~m} / \mathrm{s}$ (Alboussiere et al., 2010). However, Loper (2007) theoretically estimated the velocity amplitude of compositional plumes near the ICB, and suggested that their value could be $1.3 \times 10^{-3} \mathrm{~m} / \mathrm{s}-0.25 \mathrm{~m} / \mathrm{s}$. This suggests that the origin of elastic anisotropy in the inner core could be attributed to Joule heating.

The advantage of the present model is that the velocity amplitude in the interior of the inner core does not depend on viscosity, the value of which is quite ambiguous in the inner core. However, the present estimation may be affected by other parameters. For example, smaller toroidal magnetic field at ICB $B$ brings smaller Joule heating and then, smaller velocity amplitude. The value $10^{-1} T$ used in the present study may be rather large, since several recent studies proposed the averaged values of magnetic field of a few $\mathrm{mT}$ in the interior of the present outer core (e.g. Christensen and Aubert, 2006; Gillet et al., 2010; Buffett, 2010). The toroidal part of the magnetic field at the ICB may be significantly larger, for example, due to the differential rotation of the inner core (e.g. Aurnou et al., 1998), however, recent seismological studies yield relatively small rotation rates (e.g Tkalc̃ić et al., 2013) or infer no differential rotation (e.g Mäkinen and Deuss, 2011). The value of difference between basic and adiabatic temperature at the center also affects the estimation, which is influenced by thermal history of the inner core. When the temperature difference becomes small, the velocity amplitude increases. Thermal history of the inner core should be reexamined with a recently updated value of thermal conductivity to evaluate the temperature difference. 
ICB tends to be impermeable at $u^{\prime} \sim O\left(10^{-3}\right) \mathrm{m} / \mathrm{s}$ in our estimation. This transition turbulent velocity in the outer core $u_{c}^{\prime}$ depends on several parameters. In order to clear this issue, let us remove $h$ from the boundary conditions (13) and (14) using (22). After non-dimensionalizing these equations, we obtain,

$$
\frac{\partial}{\partial r} r\left(-\nabla^{2} \Phi+\frac{2 L_{2} \Phi}{r^{2}}\right)=-\mathcal{P}_{\eta} \frac{L_{2} \Phi}{r}, \quad \nabla^{2} \nabla^{2} \Phi=-\mathcal{P}_{T} \frac{L_{2} \Phi}{r}, \quad \text { at } r=1,
$$

206

$\mathcal{P}_{D}=\frac{\delta \rho g a}{\rho v} \cdot \frac{L}{C_{p}\left(m_{m}-m_{a d}\right) \rho g u^{\prime}}=\frac{\tau_{p}}{\tau_{\eta}}, \quad \mathcal{P}_{T}=\frac{\alpha g m_{m} \rho g a^{2}}{v} \cdot \frac{L}{C_{p}\left(m_{m}-m_{a d}\right) \rho g u^{\prime}}=\frac{\Delta T_{m}}{\Delta T_{v}}$.

$\mathcal{P}_{D}$ is the non-dimensional parameter expressing the effect of phase change on the dynamical balance at ICB (Deguen et al., 2013), meaning the ratio between the phase change timescale $\tau_{p}=L /\left[C_{p}\left(m_{m}-m_{a d}\right) \rho g u^{\prime}\right]$ and the viscous relaxation timescale $\tau_{\eta}=(\rho v) /(\delta \rho g a) . \mathcal{P}_{T}$ is the non-dimensional parameter expressing the effect of phase change on the thermal balance at ICB, interpreted as the ratio between the temperature scale induced by surface displacement $\Delta T_{m}=m_{m} \rho g \tau_{p} V$ and that induced by viscous and buoyancy forces balance $\Delta T_{v}=(\nu V) /\left(\alpha g a^{2}\right)$, where $V$ is the velocity scale. Whether ICB becomes permeable or impermeable is determined by the values of $\mathcal{P}_{D}$ and $\mathcal{P}_{T}$, When both $\mathcal{P}_{D}$ and $\mathcal{P}_{T}$ approaches 0 , instantaneous phase change occurs at and ICB becomes fully permeable. In contrast, either $\mathcal{P}_{D}$ or $\mathcal{P}_{T}$ is sufficiently large, ICB becomes impermeable due to slow phase change. Both $\mathcal{P}_{D}$ and $\mathcal{P}_{T}$ depend on several parameters, respectively. For example, if viscosity becomes large and other parameters are fixed, both $\mathcal{P}_{D}$ and $\mathcal{P}_{T}$ is reduced, resulting permeable ICB. In other words, larger viscosity gives smaller transition turbulent velocity $u_{c}^{\prime}$. Note that the conditions $\mathcal{P}_{D} \sim 1$ and $\mathcal{P}_{T} \sim 1$ and the values of the paramters used in this study give $u_{c}^{\prime} \sim 0.2 \mathrm{~m} / \mathrm{s}$ and $0.03 \mathrm{~m} / \mathrm{s}$, seeming to contradict the present numerical results. However, since the thickness of the boundary layer is about 0.2 in our solutions, the lefthandsides of Eq. (23) should not be assumed as $O(1)$ but $O\left(0.2^{3}\right)$ and $O\left(0.2^{6}\right)$, yielding $u_{c}^{\prime} \sim O\left(10^{-3}\right) \mathrm{m} / \mathrm{s}$ and $O\left(10^{-6}\right) \mathrm{m} / \mathrm{s}$, which is consistent with the numerical results.

The present results show that when the surface displacement of the inner core is significant the solid state flow is restricted to the surface of the ICB where anisotropy in the present inner core is weaker. It seems that Joule heating is unsuitable for the

where 
origin of the elastic anisotropy. However, the mechanism proposed here might play a important role in the past, possibly because heat flux through the core-mantle boundary was larger, yielding stronger magnetic field in the outer core. There is a possibility that the elastic anisotropy was produced by the solid state flow driven by Joule heating during the growing stage of the inner core, and is now buried while the mechanism is not operating (e.g. Deguen and Cardin, 2009).

\section{References}

Alboussiere, T., Deguen, R., Melzani, M., 2010. Melting-induced stratification above the Earth's inner core due to convective translation. Nature 466, 744-747.

Aurnou, J., Brito, D., Olson, P., 1998. Anomalous rotation of the inner core and the toroidal magnetic field. J. Geophys. Res., 103, 9721-9738.

Bergman, M. I., 1997. Measurements of electric anisotropy due to solidification texturing and the implications for the Earth's inner core. Nature 389, 60-63.

Buffett, B. A., 2010: Tidal dissipation and the strength of the Earth's internal magnetic field. Nature, 468, 952-954.

Buffett, B. A., Wenk, H.-R., 2001. Texturing of the Earth's inner core by Maxwell stresses. Nature 413, 60-63.

Christensen, U. R., Aubert, J., 2006. Scaling properties of convection-driven dynamos in rotating spherical shells and application to planetary magnetic fields. Gephys. J. Int., 166, 97-114.

Deguen, R., Cardin, P., 2009. Tectonic history of the Earth's inner core preserved in its seismic structure. Nature GeoScience 2, 419-422.

Deguen, R., Alboussiere, T., Cardin, P., 2013. Thermal convection in Earth's inner core with phase change at its boundary. Geophys. J. Int., 194, 1310-1334.

Gillet, N., Jault, D., Canet, E., Fournier, A., 2010. Fast torsional waves and strong magnetic field within the Earths core. Nature, 465, 77-77. 
Jeanloz, R., Wenk H.-R., 1988. Convection and anisotropy of the inner core. Geophys. Res. Lett. 15, 72-75.

Karato, S., 1993. Inner core anisotropy due to the magnetic field-induced preferred orientation of iron. Science 262, 1708-1711.

Karato, S., 1999. Seismic anisotropy of the Earth's inner core resulting from flow induced by Maxwell stresses. Nature 402, 871-873.

Loper, D. E., 2007. Turbulence and small-scale dynamics in the core. in Schubert, G. (Editor) Treatise on Geophysics 8, 187-206.

Mäkinen, A. M., Deuss, A., 2011. Global seismic body-wave observations of temporal variations in the Earth's inner core, and implications for its differential rotation. Geophys. J. Int., 187, 355-370.

Morelli, A., Dziewonski, A. M., Woodhouse, J. H., 1986. Anisotropy of the inner core inferred from PKIKP travel times. Geophys. Res. Lett. 13, 1545-1548.

Poupinet, G., Pillet, R., Souriau, A.,1983. Possible heterogeneity of the Earth's core deduced from PKIKP travel times. Nature 305, 204-206.

Pozzo, M., Davies, C., Gubbins, D., Alfe, D., 2012. Thermal and electrical conductivity of iron at Earth's core conditions. Nature 485, 355-358.

Stacey, F. D., Davis, P. M., 2008. Physics of the Earth. Cambridge University Press, 532pp.

Souriau, A., 2007. Deep earth structure - the earth's cores. in Treatise on Geophysics, ed. by Schubert, G., 655-693pp.

Takehiro, S., 2011. Fluid motions induced by horizontally heterogeneous Joule heating in the Earth's inner core. Phys. Earth Planet. Inter. 184, 134-142.

Tkalc̃ić, H., Young, M., Bodin, T., Ngo, S., Sambridge, M., 2013. The shuffling rotation of the Earth's inner core revealed by earthquake doublets. Nature Geosci., 6, 497502. 
281 Yoshida, S., Sumita, I., Kumazawa, M., 1996. Growth model of the inner core coupled with the outer core dynamics and the resulting elastic anisotropy. J. Geophys. Res. 101, 28085-28103. 